\title{
A Retrospective Study to Determine the Prevalence and Outcome of Tuberculosis among Patients Who Visited the TB Annex Hospital in Congo Town, Monrovia, Liberia from July 2009 to July 2010
}

\author{
Patrick N. Kpanyen (Corresponding author) \\ Department of Epidemiology and Biostatistics, School Of Public Health \\ Tongji Medical College of Huazhong University of Science and Technology \\ Wuhan, Hubei 430030, China
}

Tel: 86-1597-222-9538Ｅ-mail: pkpanyen@yahoo.com

Gorgui Diouf

Department of Epidemiology and Biostatistics, School Of Public Health

Tongji Medical College of Huazhong University of Science and Technology

Wuhan 430030, Hubei, China

E-mail: gidiouf@hotmail.com

Augustine F. Tokpa

Department of Epidemiology and Biostatistics, School Of Public Health

Tongji Medical College of Huazhong University of Science and Technology

Wuhan 430030, Hubei, China

E-mail: tokpa101@yahoo.com

Moses W. Toe

Cuttington University, College of Health Sciences

Gbarnga, Suakoko, Bong County, Republic of Liberia, West Africa

Shaofa Nie

Department of Epidemiogy and Biostatistics, School Of Public Health

Tongji Medical College of Huazhong University of Science and Technology

Wuhan 430030, Hubei, China

E-mail: sf_nie@mails.tjmu.edu.cn

Received: November 25, 2010 Accepted: December 14, 2010 doi:10.5539/gjhs.v3n1p110

\begin{abstract}
This study was conducted at the TB Annex Hospital in Monrovia; the capital city for Liberia. The Annex is the only public health center build currently to treat TB and its related complications. The major aim of this paper was to determine the rate of sputum positive cases among those who visited the TB Annex Hospital from July 2009 to July 2010 and factors (age, gender, education, time and season) associated with this probability. Additionally, it was meant to find the rate of treatment compliance and various factors also associated with its probability. A cross-sectional descriptive study design was used and all the 299 patients who visited the Center to seek health care's hospital charts were thoroughly reviewed. Results showed in Figure 1 that the prevalence of TB was very high among those who visited the TB Annex Hospital. The Exact Binomial Test was used with
\end{abstract}


$\mathrm{p}<0.05$ and CI $(0.864-0.934)$.Accordingly, the monthly incidence of the disease remains very high ranging from $75 \%$ to $100 \%$ respectively. Amazingly, in January 2010 alone, all 42 patients who visited the Annex were sputum positive. During the period under study according to Table 1 and looking at the sputum screening test by age distribution as a variable, the various age range were set at 0-6,7-12,13-18,19-24, 25-30and 31years and above respectively. Accordingly, the latter recorded the highest number of patients 126 out of 299 yet with $\mathrm{p}>0.05$. Out of the 299 patients, 172 was males with 127 females. For their educational level, its corresponding $\mathrm{p}$ is $<0.05$ indicating that the disease was distributed based on their level of education. The dry season recorded 133 patients out of which 124 were sputum positive cases. According to Table 2, age group 31 and above completed their TB treatment $(66.09 \%)$ and the rest closely dispersed but $\mathrm{p}>0.05$. For gender, the females did extremely well $(80.18 \%)$ in complying with treatment than their male counterparts $p<0.05$. There was no difference seen with regards to the distribution of the disease by season as a factor.

Keywords: Tuberculosis, TB Annex Hospital, Monrovia, Health sector, Grave yard cough, Heamoptysis, Caseworker, Compliance, Ministry of Health and Social Welfare

\section{Introduction}

This retrospective study to determine the prevalence and outcome of tuberculosis among patients who visited the TB Annex Hospital was conducted in Monrovia, the capital of Liberia. Liberia was founded in 1822 as an outpost for returning freed slaves from the Americas. It grew into a colony and eventually became a commonwealth, and achieved independence July 26, 1847 with the help of the American Colonization Society (a private organization based in the United States). Liberia is situated on the west coast of Africa and covers a land surface area of about $111,370 \mathrm{~km}$ with an estimated population of 3.5 million people. The climate is tropical with significant variations between the dry and wet rainy seasons. The rainy season commences in April and ends in October, while the dry season runs from November to March. Liberia experienced one of the worst civil war (1989-2003) which adversely affected all fabrics of the country's national life. The current situation in the health sector is far from satisfactory. Accordingly, the health sector experienced extensive destruction of its infrastructures, displacement of health workers for fear of their lives, massive looting and destruction of equipment, including drugs and medical supplies. It is estimated that only $16 \%$ of the country's population have access to modern health care services. There are no current and accurate statistics on the number and type of health manpower within the country, both public and private. Current estimates indicate that there are less than 1,300 professional health workers in the public health system in all professional categories. A cursory review of health manpower situation reveals that the distribution of health manpower remains skewed in favor of Montserrado County which Monrovia; the nation's capitol is also the seat. Montserrado County alone accounts for over $90 \%$ of all physicians in the country, and nearly $60 \%$ of all professional nurses and paramedical personnel $(1,2)$.

The TB germ maybe as old as the earth itself, surviving in the primeval mud at the beginning of time, Mycobacterium tuberculosis has been present in the human population since antiquity. Fragments of the spinal column from Egyptian mummies from $2400 \mathrm{BCE}$ show definite pathological signs of tubercular decay. Evidence of TB appears in Biblical scripture, in Chinese literature dating back to around $4000 \mathrm{BC}$, and religious books in India around 200BC.Physicians in ancient Greece called it "Phthisis" to reflect its wasting characteristics. In recent times, tuberculosis has been called "consumption". More than $90 \%$ of TB cases occur in developing nations like Liberia and others that have poor resources, facilities, and high number of people infected with HIV. Tuberculosis increased since 1985, most likely due to the increase in HIV and continuous to be a worldwide problem. In 2008, the World Health Organization (WHO) estimated that one-third of the global population was infected with TB bacteria. In Liberia, it is often refer to as G.Y.C. which means Grave Yard Cough. This perception negates efforts in trying to get a good prognosis out of a curable disease. There is only one TB annex hospital in the whole of Liberia with a capacity of 200beds established to treat TB cases and its related complications. The hospital is located in Congo Town, adjacent the Samuel Kanyan Doe boulevard and has a catchment population of over 20,000 persons. Of course, the Center was hard hate by the civil war and was abandoned by patients and health workers alike who fled for fear of their individual lives. There is also a leprosy colony in Ganta, Nimba County, in the north of Liberia that takes care of people with leprosy, yet sees and treats few cases of TB.

With just one TB center in the entire country, providing quality health care to the citizens is one of the major daunting challenges faced by the national government coupled with the burden of HIV/AIDS pandemic, high maternal mortality, malaria and malnutrition just to name a few. Most of the public health facilities are without essential drugs and equipment. People in rural areas have no access to confirmatory laboratory equipment and are often treated on the basis of presenting signs and symptoms for TB. The most common one being coughing 
heavy and coughing out with blood (haemoptysis).Smoking cigarette is also rule out as the most common indicator. Even if patient started the TB treatment, there may be shortage of their drug or no funds at all to purchase same and continue the course of treatment. This usually led to relapses and subsequently death from a disease that is curable. Families too make matters worse by shying away from their relative for fear of contracting same especially if the patient coughs out blood. Usually, the patient's utensils and other personal effects are separated from the normal household and a mishifit structure quickly prepared for the patient to move in and battle with his or her poor health condition. There is stigma in the community when it comes to TB patients because nobody wants to be around someone who is sick. When they see them cough, they become afraid. National government is trying to combat that stigmatization by encouraging patients to return home once they have completed the initial eight weeks treatment and can no longer spread tuberculosis to others. Communities are encouraged to embrace patients without stigmatization. At the TB annex, when patients are confirmed, they are admitted and immediately put on the first course of treatment for eight weeks and discharged home during the second phase (continuation phase), to give space to more critical patients due to lack of capacity at the annex. During the intensive phase, four drugs are taken under observation each day for two months. The patients usually take with them medication for four weeks of two medicines per day and a return to clinic date given them at a monthly interval for re-supply for a total of six months. This is also another way to check treatment compliance as well as checking related complications. But in most cases, when these patients fell like they are getting better and not critical anymore, they would totally abandon the next course of treatment.Re-examination of sputa at the end of each treatment phase is considered very important $(2,3,4)$. In Liberia, the difficulties in getting a traffic vehicle, inaccessible roads, poor funding, and the stress in walking on foot several hours to get to health service delivery areas is yet another factor for noncompliance. In this case, the families are told and encouraged to ensure that their relative(s) comply with treatment for a better prognosis; but to no avail in most cases. Against this backdrop, a strategy was developed and caseworkers trained with the help of (W.H.O) from various communities to serve as watch dogs, follow-up patients and report cases of treatment compliance and or abandonment. This too has proven unsuccessful due to lack of funds. The number of TB cases reported in Liberia is on the rise at a time when health officials say nearly $40 \%$ of cases world wide are not properly detected and treated. So, this study is aimed at determining the prevalence and outcome of patients who visited the only TB Annex Hospital in Liberia. It is only a pioneering attempt to give an overall picture of TB condition in a country just emerging from fourteen years of civil unrest.

\section{Aims of the study}

The general purpose of this study is to determine the rate of sputum positive cases among those who visited the TB Annex Hospital in Monrovia from July 2009 to July 2010 and factors (age, gender, education, time and season) associated with this probability. In addition, the study is also aimed at finding the rate of treatment compliance and factor associated with this probability among the study subjects.

\section{Materials and Methods}

\subsection{Study Population}

The study was conducted at the only TB annex hospital in the nation's capital, Monrovia, Montserrado County, Liberia. This 200 bed hospital was established to treat TB and its related complications with a catchment population of over 20,000 people. However, being the only TB Annex Hospital in the nation, people come from all walks of life in the country for screening and treatment purpose if diagnose with TB. By the use of a systematic records approach method, the medical charts for all patients who visited the TB Annex Hospital in Congo Town, Monrovia from July 2009 to July2010 were thoroughly reviewed. A total of 299 patients' charts were reportedly available and seen.

\subsection{Study Design}

A cross-sectional study design was used to form the data base for this research. In this design, there was a purposive exploration of all patients' charts available and covering the period July 2009 to July 2010 who sought medical care at the TB Annex Hospital and to subsequently arrive at conclusions about the trend and outcome of TB screening, diagnosis and treatment. In this way, it seeks to describe and explain the available records for all those patients who visited the annex either voluntarily or were brought by a family member or care taker as a unit. All the 299 patients that visited the Annex's charts, both males and females, young and old were purposely sorted out to form the data base for this inquiry. General demographic characteristics such as age, gender, educational level, occupation, geographical location, marital status among others and treatment compliance or noncompliance were observed. The charts were reviewed several times and then double checked with the help 
and supervision of the data managers of the TB Annex to verify, explain and validate certain variables when not understood by the investigator.

\subsection{Procedure}

A step by step records review method was done in which all of the patients who attended the TB annex hospital from July 2009 to July 2010 charts were consciously selected and tallied. Since this was focused information, first the years were identified and various frequencies matched and registered against the various months. A data collection form was completed that recorded each patient's personal and demographic profile. The most important variables driving the research, which were a confirmatory diagnosis with TB and beginning the initial phase of treatment were also seriously recorded. Other factors such as the most common clinical symptoms reported by patients in their chief complaint were observed and noted. Most of them reported presenting with fever, weight loss, severe cough, night sweats and coughing out with blood (haemoptysis). The charts were identify upon seeing the confirmatory diagnosis and recorded to form the basis for the investigation.

\subsection{Statistical Analysis}

The various frequencies were entered into Microsoft EXCEL for windows (version 2007), and the figure and tables all developed. Data was later transported to SPSS version 18.0, PASW Statistics 18 (SPSS Inc. Chicago, Illinois, U.S.A.) Statistics software R version 2.12.0 was also used to ascertain the results. The prevalence was calculated as well as the Chi square statistics to determine level of significance between groups and variables.

\subsection{Ethical Consideration}

This study was reviewed and approved by the ethical committee at the central Ministry of Health and Social Welfare, Republic of Liberia and the management of the TB Annex Hospital. A cross examination was carried out to ensure ethics, that the medical records left in charge of the center will be protected and information contained therein will be kept confidential. As a result of that, the many visitations made at the annex for the purpose of reviewing patients' record was done during working hours (8:00am-5:00pm) and was done under the watchful eyes of the data managers to ensure safety. The data managers themselves were very vigilant in enforcing the rules and regulations of the Center as well as the Central Ministry of health and Social Welfare of the Republic of Liberia to the latter by protecting the patients' rights.

\section{Results}

According to Figure 1 which showed the monthly clinic attendance distribution pattern as well as the various frequencies of the disease, the total number of 299 persons who visited the Annex's medical charts was reviewed. Of this number, 270 were diagnosed sputum positive for tuberculosis. The prevalence of the disease was calculated to be 0.90 among those who visited the Annex. The Exact Binomial Test was used to determine the level of significance at 0.05 and the number of successes and trials put into statistical software $\mathrm{R}$ version 2.12.0. The result was a p-value $<0.05$ with a $95 \%$ CI $(0.864-0.934)$. This showed a significantly high prevalence. Additionally, various monthly frequencies were also calculated beginning from July 2009 to July 2010 using the same software. In July 2009, 20 out of 25 patients had positive sputa for tuberculosis which is $80 \%$, August 16 out of $20(80 \%)$, September, 13 out of $15(86 \%)$, October 18 out of $19(95 \%)$, November 9 out of $12(75 \%)$ and December 17 out of $18(94 \%)$ respectively. In 2010 beginning with January all 42 patients were sputum positive (100\%), February 37 out of 39 (94\%), March 19 out of 22 (86\%), April 13 out of $15(86 \%)$, May 25 out of 27 (92\%), June 20 out of $23(86 \%)$ and July 21 out of 22 (95\%). According to Table 1 which showed the sputum TB test outcome among those who visited the TB Annex Hospital from July 2009 to July 2010 by age, gender, education, and season, age range 0-6 years was not observed. A total number of 299 patients reported out of which 270 were tested sputum positive while 29 were sputum negative. The table further show that age range $7-12$ recorded $20(90.91 \%)$ out of 22 patients being sputum positive while the other $2(9.09 \%)$ patients were negative, 13- 18 years $31(88.57 \%)$ out of 35patients sputum positive and $4(11.43 \%)$ sputum negative, 19-24 years $48(90.57 \%)$ out of 53 sputum positive while $5(9.43 \%)$ were sputum negative. Age range 25-30years had a frequency of 63 out of which $56(88.89 \%)$ were sputum positive leaving $7(11.11 \%)$ as sputum negative. Age range 31 and above had the highest recording of 126 patients out of which115 (91.27\%) were sputum positive and $11(8.73 \%)$ sputum negative, comparing the sputum test outcome by age as a factor, there was no difference observed, henceforth, a $\chi^{2}$ of $0.4116 \mathrm{p}>0.05$. Using the same sputum test outcome by gender, there were 172 males out of which $159(92.44 \%)$ were sputum positive leaving $13(7.56 \%)$ as sputum negative. As for the women, there was a total of 127 out of which $111(87.40 \%)$ were sputum positive while $16(12.60 \%)$ were sputum negative $\mathrm{p}>0.05$. The third factor consider was education in which those with out formal education presented high with 84 patients out of which $75(89.29 \%)$ were sputum positive and 9 (10.71\%) negative, elementary school 77 out of which $71(92.21 \%)$ were positive and $6(7.79 \%)$ sputum negative. Junior high 
school 61(95.31\%)sputum positive out of 64 and $3(4.69 \%)$ sputum negative, high school $56(90.32 \%)$ out of 62 as sputum positive and $6(9.68 \%)$ sputum negative. Those with College education were just 12 out of which 7 $(58.33 \%)$ were sputum positive and $5(41.67 \%)$ sputum negative, hence $\chi^{2} p<0.05$.Regarding season as a factor, the Dry season recorded 133 patients out of which 124 (93.23\%) were sputum positive and $9(6.77 \%)$ sputum negative while the number of patients in the rainy season were 166 out of which $146(87.95 \%)$ were sputum positive and $20(12.05 \%)$ negative, henceforth $\chi^{2} \mathrm{p}>0.05$.

According to Table 2 which showed the status and compliance rate of the patients who visited the TB Annex Hospital from July 2009 to July 2010 by factors such as age, gender, education and seasonal distribution pattern of the disease, 270 out of 299 patients were tested sputum positive. Looking at their status and compliance rate, age range range 0 -6years was not observed. Accordingly, those 7-12 years were 20 out of which $12(60 \%)$ completed treatment, $5(25 \%)$ did not complete, and $3(15 \%)$ were reported dead. For age range 13-18years, a total of 31patients were sputum positive out of which 17 (54.84\%) completed treatment, $13(41.94 \%)$ did not complete and $1(3.23 \%)$ dead reportedly, age range 19-24years recorded 48patients out of which24 (50\%) completed treatment, 20(41.67\%) did not complete and $4(8.33 \%)$ dead. The highest frequency was age range 31 and above years with 115 patients out of which 76 (66.09\%) completed treatment, $24(20.87 \%)$ did not and 15 (13.04\%) reported dead, hence $\chi^{2} \mathrm{p}>0.05$. Using gender as a variable, a total of 159 males were recorded out of which $70(44.03 \%)$ completed treatment while72 (45.28\%) did not complete 17(10.69\%) dead. Females reported a total of 111 patients out of which $89(80.18 \%)$ completed treatment, $13(11.71 \%)$ did not complete and $9(8.11 \%)$ dead, $\chi^{2} \mathrm{p}<0.05$. This showed a difference in the compliance rate using sex as a variable. Looking at education, patients without formal education, $38(50.67 \%)$ completed treatment, $26(34.67 \%)$ did not complete and $11(14.6 \%)$ reported dead making a total of 72patients.Elementary school 39 (54.93\%) completed treatment, 25 (35.21\%) did not complete, 7 (9.86\%) dead making a total 71. Junior high school, 36 (59.02\%) completed treatment, 21 (34.43\%) did not complete, and 4(6.56\%) dead making a total of 61while high school 40(71.43\%) completed treatment, $13(32.21 \%)$ did not complete and 3(5.36\%) dead making a total 56. For their college counterparts, out of a total of 7, $6(85.71 \%)$ completed treatment, none of them forfeited, but $1(14.29 \%)$ reported dead. Looking at season as a factor, 124 patients were tested sputum positive out of which $76(61.29 \%)$ completed treatment, $39(31.45 \%)$ did not and $9(7.26 \%)$ dead reportedly during the dry season. In the rainy season, 146 patients tested sputum positive. Of that number, $83(56.85 \%)$ completed treatment, $46(31.51 \%)$ did not complete and $17(11.64 \%)$ dead reportedly with $\chi^{2} \mathrm{p}>0.005$.

\section{Discussion}

Control of tuberculosis is achieved by minimizing the period of time that cases of pulmonary tuberculosis are infective to their family and friends. This is accomplished through early detection and diagnosis of infective cases and then ensuring that patients complete a course of multidrug therapy (5). According to results presented in Figure 1, the prevalence of tuberculosis among those who visited the TB Annex Hospital from July 2009 to July 2010 was so high (0.90) and has shown a statistical significance $\mathrm{p}<0.05$ using the Exact Binomial Test. This could probably be due to the fact that most of these patients were already sick and started presenting with clinical signs and symptoms for the disease before going to the center. Accordingly, routine medical check-up in Liberia is still far-fetch and something view by most people as proving one's financial strength or waste of resources. Majority of those who go to hospital for health care go when it is very late and their individual immune system can not withstand the pathogen burden and they look so sick and in a deplorable condition. Majority of the poor ill people can not even afford the basic need for themselves, what much more about getting transportation fare to health service provision centers and paying for their medical bills. The results also show that adult patients particularly those above twenty reported the most. It is at this point of time in life in that country that an individual take full responsibility of his or herself to earn positive living. As a result, they would engage in doing hard odd jobs that exposes them to varying environmental conditions especially if they are not learn thereby increasing their risk for communicable diseases. Pervasive poverty with more than three-quarters $(76.2 \%)$ of the population living on less then USD 1.00 a day and over half (52\%) living in abject poverty with less than USD 0.50 a day give rise to communicable disease situation including TB. Liberia's current debt stands at US 3.5 billion (both external and domestic) with formal sector employment at $20 \%$ of the total employment. Housing stocks are very limited in both rural and urban areas (particularly rural areas).The housing and shelter stock, basic infrastructures and public buildings were extensively looted and damaged on account of the war. This destruction of the country's limited housing stocks coupled with massive displacement of population have compelled families to reside in mishifit structures and displaced camps. Access to safe drinking water is still a major problem in the country. In 2000 , approximately $32 \%$ of the population had access to safe drinking water with urban access being $55 \%$ as compare to $10 \%$ in rural areas. A greater portion of the population continuous to 
source drinking water from tubes wells and bore holes sometimes fitted with pumps or get water from streams and river banks. The capital, Monrovia and adjacent areas depend on water supplied by tankers. The situation with respect to sanitation is even worse. In 2000 , only $36 \%$ of households had access to proper sanitation; $71 \%$ of urban dwellers compare to $18 \%$ of rural dwellers. The remaining $64 \%$ of households continue to dispose of their human waste in bushes, streams, rivers, ponds, beaches, and anywhere else in the communities that they find convenient. These conditions of course favor the emergence of communicable diseases like TB and others. The acute shortage of health facilities and even access to health care make matters worse. In 2002, there were 346 public sector health facilities. Of this number, 18 were hospitals, 37 health centers, and 291 clinics (to be verified). Though these figures are yet to be verified, however, not all of these facilities are functioning. Close to $70 \%$ are nonfunctional. Nearly all of these facilities are in the state of despair. In the private sector too, there were 187 facilities disaggregated as follows; 10 hospitals, 10 health centers and 67 clinics (to be verified) (2, 4, 6). In this study, it was observed that majority of the patients were adult males, the reason is apparent in that males are the ones who are consider bread winners for the family. They are the ones who do the odd jobs and engage in other risk behaviors that jeopardize their health more than women. In the same token, males would usually forfeit treatment of this kind for it is well established culture that men are able to endure any thing awkward than women. As a result, men usually keep to their busy schedule and downplay their poor health conditions. You would also notice that compliance rate was better for children because the mothers would usually exhibit a positive attitude to help their ill child than men especially if they observed proper birth spacing and other family planning methods that can give them a manageable number of kids without stress.

Our results showed a significant difference with regards to the people's educational level. Data from Liberia indicate that despite a compulsory education law in 1934 and revised in 2001, the nation's literacy rate remains very low at $26 \%$. Current illiteracy is staggering at $63 \%$; with females accounting $26 \%$ as compared to $50 \%$ for males. Gross enrollment ratio nationally is $62 \%$ or 745,859 students. The distribution of enrollment by grade level is $45 \%$ in preschool, $71 \%$ in primary school, $6 \%$ in secondary school and $1 \%$ in vocational school and post secondary programs. Statistics on enrollment at higher institutions of learning though scanty, indicate an increase between 12,000 to 14,000 .Our results indicated that patients who are educated had a better compliance rate than those with no formal education. The reason is that most of these people were able to discover through learning that compliance means "hope for life". Therefore, they invested most of their time in completing their treatment to lower their chances for death. The result from many other countries about a person's educational level, wealth and the distribution of diseases remains unclear. Similar study in Zambia and Kenya show how poverty enhances the prevalence of diseases. In both countries, it shows that years of education significantly lower the probability of mortality of non-poor men in Kenya $(7,8)$. These findings are somewhat consistent with (de Walrus,2004) who found that over time, susceptibility to TB and HIV in Uganda declined for relatively well educated people more than poorly educated people as a measure regarding precautionary measure of spread. This could meanly be due to the fact that non-poor men and women are able to process public health messages and that education and relative wealth empowered them to translate their knowledge into practice. Link and Phelan 1995; Deaton 2002 indicates that differences across individuals in educational and economic resources will maintain a gradient in health when ever there exists a mechanism or technology that more economically better-off and knowledgeable people can access to improve their health. In Zambia, there appears to be little correlation between indicators of individual and household wealth and the probability of disease related mortality. Nor there is a significant relationship between individual education attainment and mortality for either poor or non-poor men or women $(9,10)$.

Liberia leads among its regional neighbors when it comes to some of the least impressive figures regarding health care workforce and the worst morbidity and mortality statistics. Although Liberia's long civil war left its health care service delivery system fragmented, severely damaged, and heavily dependent on international donors and Non Governmental Organizations (NGOs), in only a few years, Liberia has taken bold steps to transition from an emergency relief model of health services delivery struggling towards a functioning, decentralized health service model. The challenges are daunting in the face of many postwar reconstruction plans and strategies couple with the closure of most of the emergency health care missions. There is generally lower level of primary health care and less access to care all over Liberia especially in rural areas. World Health Organization (WHO) is helping to improve laboratory services and train TB caseworkers. But budget constraints mean treatment is limited to about 5,000 patients a year. Stigmatizing those with the disease is one of the obstacles hindering program success. Deddeh-Bah-Kesely manages Liberia's Leprosy Colony that also screen and treat TB cases. She says one of the reasons why the disease often goes undetected is because people fear being stigmatized if they are diagnosed with TB. Gabriela Adao, a Brazilian nurse that work for Medicines Sans Frontiers (MSF) emergency relief efforts for health care delivery in Liberia also made her observation about 
stigmatization in an interview. "There is still a huge stigma attached to TB and many parents refuse to admit that their child is infected with TB. They think that TB is only an adult disease. Even the national health policy regarding TB does not include children. This she noted makes it difficult to discuss with care givers. She said with respect to treatment compliance for children, it is their care givers usually the mothers who are charged with the administration of treatment properly. The main threat was that the parents lack money to pay transportation fare to come back to the clinic on a regular basis. Additionally, mothers and caregivers have more than one child and spending time and energy to travel to clinic means she has to find ways for the other children to be cared for in the meantime. Mothers and caregivers are sometimes quite sick themselves, so it's difficult for them to take care of the treatment for their sick child". World Health officials meeting in Brazil say more and more people are dying from tuberculosis than ever before especially due to the synergistic and lethal marriage relationship between HIV/AIDS as co-infections with severe fatal consequences $(11,12,13)$. Nearly half of the countries most affected are in Africa. The disease is particularly hard to fight because of the emergence of multi-drug-resistance and extensive- drug resistant-strains that are more expensive to treat. Health officials warned that there must be coordinated approach to a disease that annually kills more than 1.5 million people in the world in order to meet the millennium Development Goal (MDG) of revising the spread of TB in the next six years $(14,15,16)$.

\section{Conclusion}

Emerging from a prolonged state of war, Liberia continues to be faced with a number of daunting challenges. The high prevalence of TB (0.90) among those who visited the TB Annex Hospital during the period under review raises more questions than answers. However, it is belief to be due to the fact that most of these people started presenting with various clinical features before visiting the Annex. The treatment compliance rate was better among females than males. The trend of education as a factor show better compliance among those educated than those without education. There was no significance difference observed during the seasons of the year and the various ages range as factors for the probabilities under spotlight. The stigma attached to TB as well as some of the nomenclatures given are serious impediment for under reportage as well as under utilization of health service centers. Community participation in health planning and development to say the least is rather minimum if not lacking due to the dormancy of services in most parts of the country. Research data remains scanty and an intensive KAP (Knowledge, Attitude and Practice) study is hereby proposed at the national level were many subjects can be recruited and included in a more comprehensive research to know the actual TB situation in Liberia, a country just emerging from fourteen years of civil war.

\section{Acknowledgements}

The authors say thank you very much to the staff and Ethics Committee of the Central Ministry of Health and Social Welfare and the management of the TB Annex Hospital, Republic of Liberia for allowing them access to this useful data. Special recognition is giving to the trained data managers of the Annex for their supervisory role by providing, and making verifications were possible. Thanks also to Dr. Henrique Tokpa and the Administration of Cuttington University, Liberia, for providing funding that facilitated the data collection. We are indebted to all those who assisted usone way or the other in making this work a success. We thank you all.

\section{References}

Corbett EL, Watt CJ, Walker N, Maher D, Williams BG, Raviglione MC, et al. (2000). The growing burden of tuberculosis: global trends and interactions with the HIV epidemic. Arch Intern Med 2003; 163: 1009 Demography Health Survey (DHS), Monrovia, Liberia: Government of Liberia.

Gillespie,S.R., S. Kadiyala, and R. Greener. (2007). Is Poverty or Wealth Driving HIV Transmission? AIDS.21, Supplement 7:S5-16.

Glyne R.J., M. Carael,A. Buve, S. Anagonou, L.Zekeng, M. Kahindo, and R. Musonda. (2004). Does Inceased General Schooling Protect against HIV Infection? Astudy in four cities. Tropical Medicine and International Health, 9.1:4-14.

Gothi D, Joshi JM. (2004). Clinical and laboratory observations of tuberculosis at a Mumbai (India) clinic. Postgrad Med J, 80: 97-100.

Gregson, S., H. Waddell, and S. Chandiwana. (2001). School Education and HIV Control in Sub-Saharan Africa: From Discord to Harmony? Journal of International Development, 13: 467-85.

Hargreaves, J.R. and J. Glynn. (2002). Education Attainments and HIV-1 Infection in Developing Countries: A systematic Review. Tropical Medicine and International Health, 7.6: 489-98. 
Health and Social Welfare Ministry/ Planning and Economic Affairs Ministry 2002 Report, Republic of Liberia.

Kirimi, L., W. (2008). Essays on Disease-Related Working-Age Adult Mortality: Evidence from Rural Kenya. Unpublished PhD Dissertation, Michigan State University.

Link, Bruce G. and Jo C. Phelan. (1995). Social Conditions as Fundamental Causes of Disease. Journal of Health and Social Behavior Extra Issue, 80-94.

Mesfin, S.M. and C. Schooler. (2002). Causal Connections between Socio-Economic Status and Health: Reciprocal Effects and Mediating Mechanisms. Journal of Health and Social Behavior, 43. 1: 22-41.

Narain JP, Tripathy SP, Pontali E. (2002). Tuberculosis and HIV infection. In: Narain JP, editor. Tuberculosis: epidemiology and control. New Delhi: World Health Organization Regional Office for South-East Asia.

Pritchett, L. and L. Summers. (1996). Wealthier is Healthier. Journal of Human Resources 31. 4: 841-68. Sharma SK, Mohan A. Co-infection of human immunodeficiency virus (HIV) and tuberculosis: Indian perspective. Indian J Tuberculosis, 2004; 51: 5-16.

Sudre P. (1993). Tuberculosis control in Somalia. Geneva: WHO; (EM/TUB/180/E/R/5.93).

WHO. (2003). The World Health Report 2003: Shaping the Future. Geneva; World Health Organization.

World Bank. (1999). Confronting AIDS: Public Priorities in a Global Epidemic. Revised Edition. New York: Oxford University Press.

Table 1. Sputum test status for all the patients who visited the TB Annex Hospital in Monrovia from July 2009 to July 2010 by age, gender, education and distribution by season

\begin{tabular}{|c|c|c|c|c|c|}
\hline \multirow{2}{*}{ Variable } & \multicolumn{5}{|c|}{ Sputum Test } \\
\hline & Positive & Percentage & Negative & Percentage & Total \\
\hline \multicolumn{6}{|l|}{ Age } \\
\hline $0-6$ & 0 & $\mathrm{NaN}$ & 0 & $\mathrm{NaN}$ & 0 \\
\hline $7-12$ & 20 & $90.91 \%$ & 2 & $9.09 \%$ & 22 \\
\hline $13-18$ & 31 & $88.57 \%$ & 4 & $11.43 \%$ & 35 \\
\hline $19-24$ & 48 & $90.57 \%$ & 5 & $9.43 \%$ & 53 \\
\hline $25-30$ & 56 & $88.89 \%$ & 7 & $11.11 \%$ & 63 \\
\hline 31\&above & 115 & $91.27 \%$ & 11 & $8.73 \%$ & 126 \\
\hline \multicolumn{6}{|l|}{ Gender } \\
\hline Male & 159 & $92.44 \%$ & 13 & $7.56 \%$ & 172 \\
\hline Female & 111 & $87.40 \%$ & 16 & $12.60 \%$ & 127 \\
\hline \multicolumn{6}{|l|}{ Education } \\
\hline No formal education & 75 & $89.29 \%$ & 9 & $10.71 \%$ & 84 \\
\hline Elementary school & 71 & $92.21 \%$ & 6 & $7.79 \%$ & 77 \\
\hline Junior high school & 61 & $95.31 \%$ & 3 & $4.69 \%$ & 64 \\
\hline High school & 56 & $90.32 \%$ & 6 & $9.68 \%$ & 62 \\
\hline College & 7 & $58.33 \%$ & 5 & $41.67 \%$ & 12 \\
\hline \multicolumn{6}{|l|}{ Season } \\
\hline Dry & 124 & $93.23 \%$ & 9 & $6.77 \%$ & 133 \\
\hline Rainy & 146 & $87.95 \%$ & 20 & $12.05 \%$ & 166 \\
\hline
\end{tabular}


Table 2. Status and treatment compliance rate among sputum positive cases who visited the TB Annex Hospital in Monrovia from July 2009 to July 2010 by age, gender, education and season

\begin{tabular}{ccccc}
\hline \multirow{2}{*}{ Variable } & \multicolumn{3}{c}{ Status and Compliance Rate } \\
\cline { 2 - 4 } Age & Completed Treatment & $\begin{array}{c}\text { Did not complete } \\
\text { treatment }\end{array}$ & Reported Dead & Total \\
$0-6$ & $0(\mathrm{NaN})$ & $0(\mathrm{NaN})$ & $0(\mathrm{NaN})$ & 0 \\
$7-12$ & $12(60 \%)$ & $5(25 \%)$ & $3(15 \%)$ & 20 \\
$13-18$ & $17(54.84 \%)$ & $13(41.94 \%)$ & $1(3.23 \%)$ & 31 \\
$19-24$ & $24(50 \%)$ & $20(41.67 \%)$ & $4(8.33 \%)$ & 48 \\
$25-30$ & $30(53.57 \%)$ & $23(41.07 \%)$ & $3(5.36 \%)$ & 56 \\
$31 \&$ above & $76(66.09 \%)$ & $24(20.87 \%)$ & $15(13.04 \%)$ & 115 \\
Gender & & & & \\
Male & $70(44.03 \%)$ & $72(45.28 \%)$ & $17(10.69 \%)$ & 159 \\
Female & $89(80.18 \%)$ & $13(11.71 \%)$ & $9(8.11 \%)$ & 111 \\
& & & \\
Education & & & $11(14.67 \%)$ & 75 \\
No formal & $38(50.67 \%)$ & $26(34.67 \%)$ & $7(9.86 \%)$ & 71 \\
education & $39(54.93 \%)$ & $25(35.21 \%)$ & $4(6.56 \%)$ & 61 \\
Elementary school & $36(59.02 \%)$ & $21(34.43 \%)$ & $3(5.36 \%)$ & 56 \\
Junior high school & $40(71.43 \%)$ & $13(23.21 \%)$ & $1(14.29 \%)$ & 7 \\
High school & $6(85.71 \%)$ & $0(0 \%)$ & & 124 \\
College & & & $9(7.26 \%)$ & 146 \\
Season & & & $17(11.64 \%)$ & \\
Dry & $76(61.29 \%)$ & $39(31.45 \%)$ &
\end{tabular}

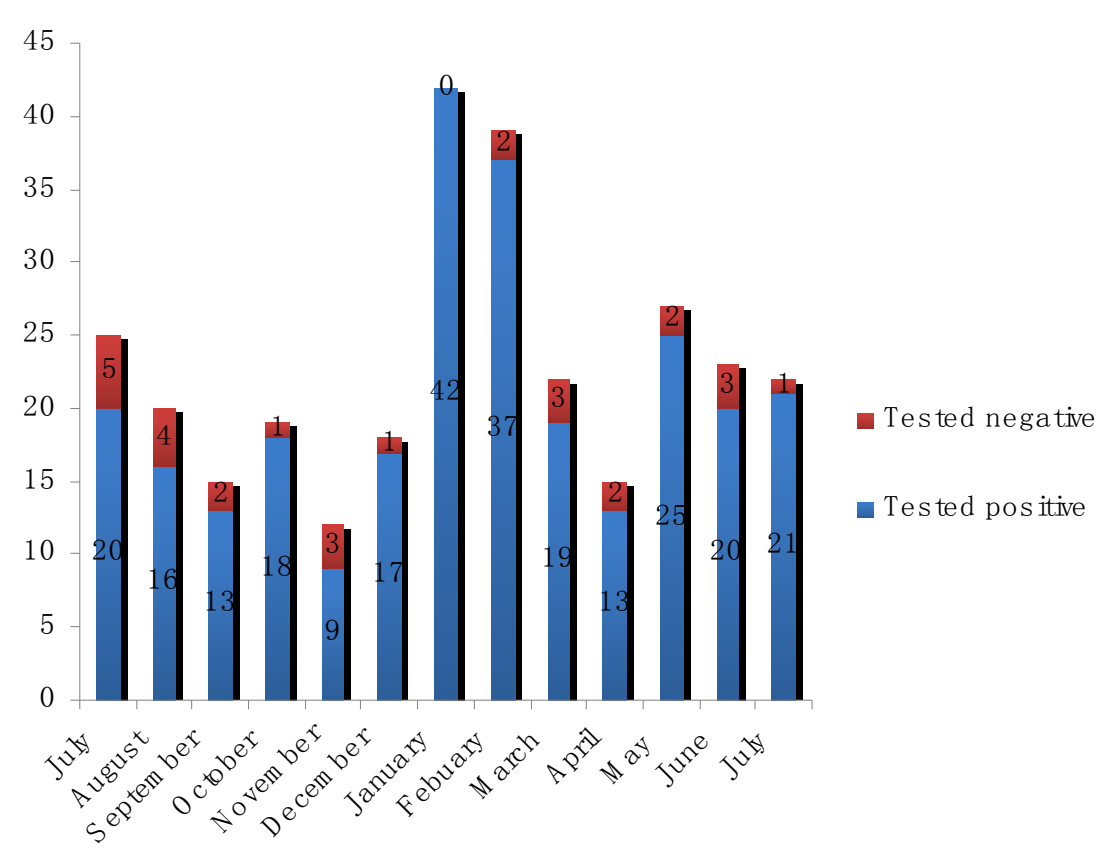

Figure 1. Total number of patients who visited the TB Annex Hospital monthly from July 2009 to July 2010 and their and respective TB screening test result 